\title{
Laser-Induced Fluorescence Velocity Measurements in Supersonic Underexpanded Impinging Jets
}

\author{
Jennifer A. Inman ${ }^{1}$, Paul M. Danehy ${ }^{2}$, Brett Bathel ${ }^{3}$, David W. Alderfer ${ }^{4}$, and Robert J. Nowak ${ }^{5}$ \\ NASA Langley Research Center, Hampton VA, 23681-2199
}

\begin{abstract}
We report on an application of nitric oxide (NO) flow-tagging velocimetry to impinging underexpanded jet flows issuing from a Mach 2.6 nozzle. The technique reported herein utilizes a single laser, single camera system to obtain planar maps of the streamwise component of velocity. Whereas typical applications of this technique involve comparing two images acquired at different time delays, this application uses a single image and time delay. The technique extracts velocity by assuming that particular regions outside the jet flowfield have negligible velocity and may therefore serve as a stationary reference against which to measure motion of the jet flowfield. By taking the average of measurements made in 100 single-shot images for each flow condition, streamwise velocities of between -200 and $+1,000 \mathrm{~m} / \mathrm{s}$ with accuracies of between 15 and $50 \mathrm{~m} / \mathrm{s}$ are reported within the jets. Velocity measurements are shown to explain otherwise seemingly anomalous impingement surface pressure measurements.
\end{abstract}

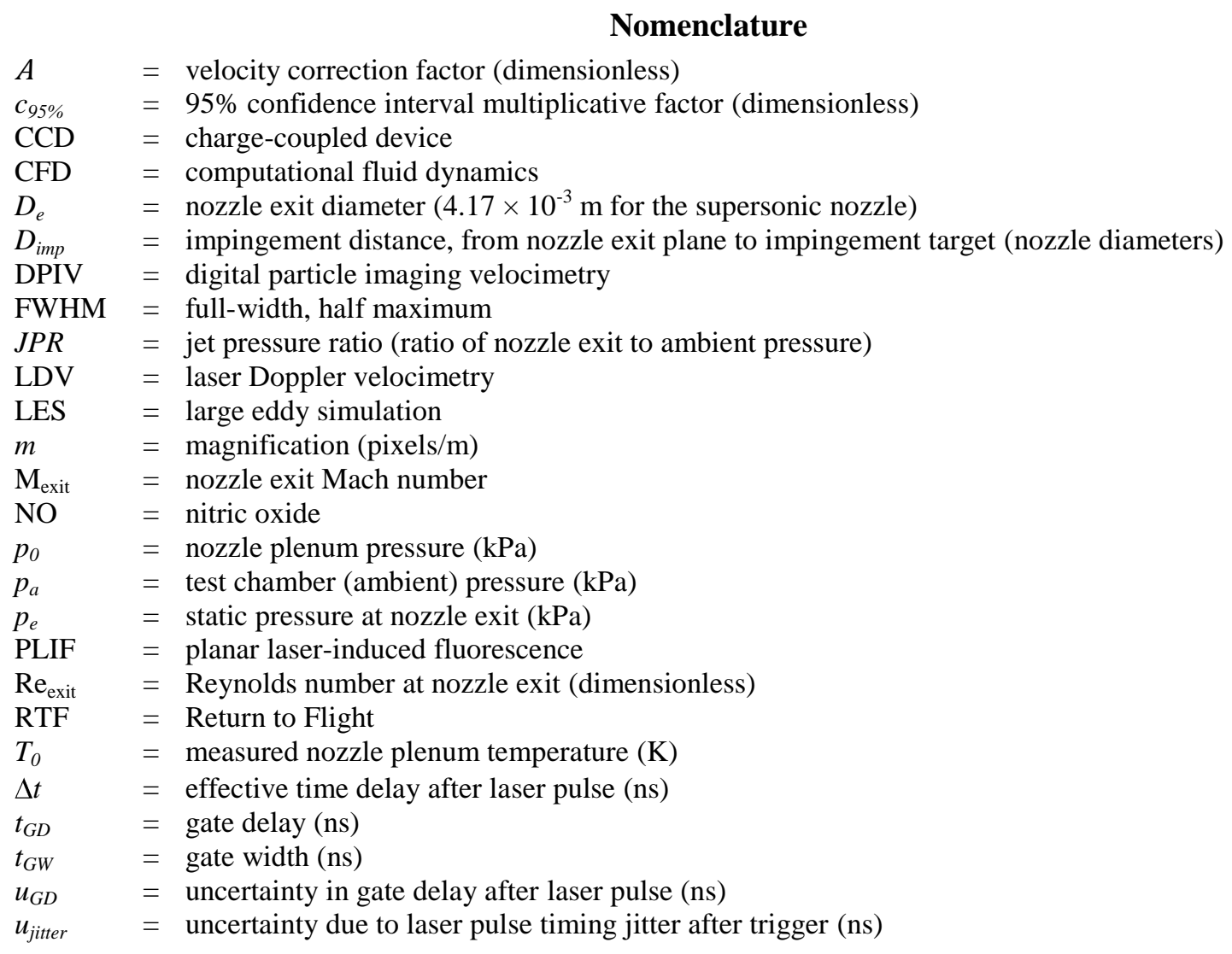

\footnotetext{
${ }^{1}$ Research Scientist, Advanced Sensing and Optical Measurement Branch, MS 493, AIAA Member.

${ }^{2}$ Research Scientist, Advanced Sensing and Optical Measurement Branch, MS 493, AIAA Associate Fellow.

${ }^{3}$ Graduate Student, University of Virginia and National Institute of Aerospace (NIA), Hampton Virginia, AIAA Student Member

${ }^{4}$ Research Scientist, Advanced Sensing and Optical Measurement Branch, MS 493.

${ }^{5}$ Distinguished Research Associate, Aerothermodynamics Branch, MS 408A, AIAA Member.
} 


$\begin{array}{ll}u_{m a g, 1} & =\text { uncertainty in magnification for regions covered by registration dotcard (pixels } / \mathrm{m}) \\ u_{m a g, 2} & =\text { uncertainty in magnification for regions not covered by registration dotcard (pixels } / \mathrm{m}) \\ u_{\Delta t} & =\text { uncertainty in effective time delay due to variable fluorescence lifetime }(\mathrm{ns}) \\ V_{e} & =\text { velocity at nozzle exit }(\mathrm{m} / \mathrm{s}) \\ \Delta x & =\text { displacement }(\mathrm{m}) \\ \theta_{i m p} & =\text { impingement angle }\left(^{\circ}\right) \\ \mu_{e} & =\text { dynamic viscosity at nozzle exit }(\mathrm{Pa} \cdot \mathrm{s}=\mathrm{kg} /(\mathrm{m} \cdot \mathrm{s}) \\ \rho_{e} & =\text { density at nozzle exit }\left(\mathrm{kg} / \mathrm{m}^{3}\right) \\ \tau_{L I F} & =\text { fluorescence lifetime, low-quenching limit }(\mathrm{ns}) \\ \chi_{N O} & =\text { mole fraction of nitric oxide (dimensionless) }\end{array}$

\section{Introduction}

This paper describes velocity measurements in an experiment designed to simulate the flow through a breach in the external surface of a supersonic aerospace vehicle and the subsequent impingement of the flow on internal structures. The tests were conducted in support of the Orbiter Aerothermodynamics Working Group as part of NASA's Shuttle Return to Flight (RTF) effort. Specifically, they were Tier I of a three-tiered program. Tier I involved using planar laser-induced fluorescence (PLIF) of nitric oxide (NO) to visualize flow issuing from a nozzle into a low-pressure chamber and then to measure the flow velocity using a time-of-flight method. Up to 25 simultaneous profiles of the axial velocity were obtained using this method. Time-averaging was used to obtain higher velocity measurement accuracy of laminar impinging jet flows. Single-shot velocity measurements were used in unsteady flows. The data are intended for validation of computational fluid dynamics (CFD) codes. Tier II encompassed using an operating wind tunnel (instead of a low-volume plenum) to supply the high temperature, high pressure gas upstream of both sonic and supersonic nozzles, with the resulting jet flow issuing into an enclosed box. Discrete pressure taps and phosphor thermography were employed to measure impingement pressures and surface heat flux. ${ }^{1}$ Tier III attempted to create more realistic flow conditions which were relevant to the vehicle breach boundary layer flow ingestion scenario with subsequent impingement on internal structures by placing an angled flat plate with various hole geometries into a wind tunnel. A box attached to the back side of the flat plate surrounded an impingement target. Surface heat flux and pressure measurements were made on separate targets using phosphor thermography, thin film gauges, and pressure taps. ${ }^{2}$

Several earlier papers by our group have reported Tier I results. Reference 3 characterizes the transition to turbulence in free (non-impinging) underexpanded sonic jets. Reference 4 compares three, free-jet, steady laminar, sonic nozzle cases with computational fluid dynamics (CFD). Reference 5 explores the relationship between flow structures and quantitative impingement surface pressure measurements for steady laminar, impinging, supersonic nozzle cases. Reference 6 identifies instability modes of transition and the importance of flow structures in contributing to flow unsteadiness for free-jet, supersonic nozzle cases. Reference 7 covers unsteady impinging jet behavior. This paper will present quantitative velocity measurements in supersonic nozzle impinging jet flows as well as comparisons with quantitative centerline impingement surface pressures.

NO PLIF was chosen to investigate this flowfield for a number of reasons. Both flow-visualization and quantitative velocimetry were desired. Schlieren flow visualization is generally simpler to implement, but was found to provide poor contrast at the low-density conditions relevant to vehicle breach flow along the atmospheric reentry profile of hypersonic vehicles. As a path-averaged technique, it also is not ideally suited for studying modes of transition to turbulence since it is unable to resolve individual turbulent flow structures, which was a primary goal of Tier I testing. Particle image velocimetry (PIV) and laser Doppler velocimetry (LDV) were also found to be illsuited to these tests due to low density flow conditions and the accompanying concerns about particles not following the flow through shock and expansion waves. Another concern was the incompatibility of the facility with the need to seed particles into the flow. In addition, LDV and hotwire anemometry were not deemed to be suitable because of the single-point nature of the techniques when velocity measurements were desired throughout an entire plane for each flow condition. The low density conditions were also found to be ill-suited for Rayleigh scattering, where the signal generated with the available laser systems would have been too low for the single-shot imaging needed to study transitional flows. In contrast, NO PLIF flow tagging velocimetry was found to be well-suited to the problem, having adequate spatial and temporal resolution along with adequate signal levels for the anticipated range of flow conditions.

One common drawback to the flow-tagging technique is that relatively short fluorescence lifetimes (typically 100-200 ns for nitric oxide, NO) mean a significant loss of signal intensity for long delays between laser firing and 
image acquisition. Fluorescence intensity exhibits a characteristic exponential decay as a function of time; consequently, the signal decay has a half-life of $t_{1 / 2}=\tau_{\mathrm{LIF}} \cdot \ln (2)$, and there will be $37 \%$ of the original signal intensity remaining after one lifetime. Since the delay must be fairly short (on the order of $500 \mathrm{~ns}$ ) to have a measurable signal, the gas velocity must be high enough to produce detectable shifts in that amount of time.

For these experiments, the NO could be seeded into a nitrogen flow, significantly reducing the quenching by oxygen encountered in air flows. Low quenching and low pressures both contributed to relatively long fluorescence lifetimes (approximately 140-190 ns). Additionally, the velocities were expected to be high enough that the gas would move several pixels during the delay between laser firing and image acquisition. (With an effective delay time of $822.5 \mathrm{~ns}$ and a magnification of $\sim 6.8$ pixels $/ \mathrm{mm}$, a velocity of $180 \mathrm{~m} / \mathrm{s}$ would produce a shift of about 1 pixel.) Recent work by Hsu et al. ${ }^{8}$ contains an excellent review of the relative advantages and limitations of competing techniques and an overview of other NO molecular tagging velocimetry (MTV) work. In addition, they explore the advantages and disadvantages of two different approaches to NO MTV measurements for obtaining twocomponent velocity maps in a variety of quenching environments in underexpanded free-jet flowfields.

\section{Experimental Methods}

\section{A. Facility and Hardware}

Tests were conducted at NASA Langley Research Center using the test section of the 15-Inch Mach 6 Wind Tunnel as a vacuum chamber. Nitrogen or helium seeded with $0.5 \%$ nitric oxide was plumbed into a heated, insulated stainless steel plenum, through a nozzle, and into the vacuum chamber. Two different nozzles were used. The first was a converging nozzle with the exit at the smallest diameter, or throat, and hereafter referred to as the "sonic" nozzle. It had a nominal exit Mach number of 1 with an exit diameter of $D_{e}=2.41 \mathrm{~mm}(0.095 \mathrm{in}$.). The second - a converging/diverging nozzle, hereafter called the "supersonic" nozzle—had a nominal exit Mach number of 2.6 with an exit diameter of $D_{e}=4.17 \mathrm{~mm}$ (0.164 in.). Mass flow controllers controlled the flow rates, which indirectly controlled the plenum pressure upstream of the nozzle. Only cases with the supersonic nozzle are discussed in this work.

A $102 \mathrm{~mm}$ (4 in.) diameter impingement disk was positioned at various distances and angles downstream of the nozzle exit. Schematics of the plenum, supersonic nozzle, and impingement disk are shown in Fig. 1. Detailed coordinates for the shape of the supersonic nozzle can be found in the Appendix in Table A1. Impingement distance (measured from the nozzle exit to the center of the impingement target along the jet axis) was varied from $25.4 \mathrm{~mm}$ (1.0 in.) to $127 \mathrm{~mm}\left(5 \mathrm{in}\right.$.). Impingement angle was defined to be $90^{\circ}$ when the impingement disk was oriented parallel to the plane of the nozzle exit, which is also normal to the jet axis. At the center of this disk was an inner 2inch diameter disk instrumented with 32 pressure taps. They were spaced $1.14 \mathrm{~mm}(0.045 \mathrm{in}$.) apart, and had an inside diameter of $0.53 \mathrm{~mm}(0.021 \mathrm{in}$.). The taps were oriented in a vertical plane (which was also the plane of the laser sheet), on the jet centerline. From the camera's viewing angle, the jet flow was from left to right. Flow visualization and pressure measurements acquired with this same apparatus have been previously reported. ${ }^{4-7}$

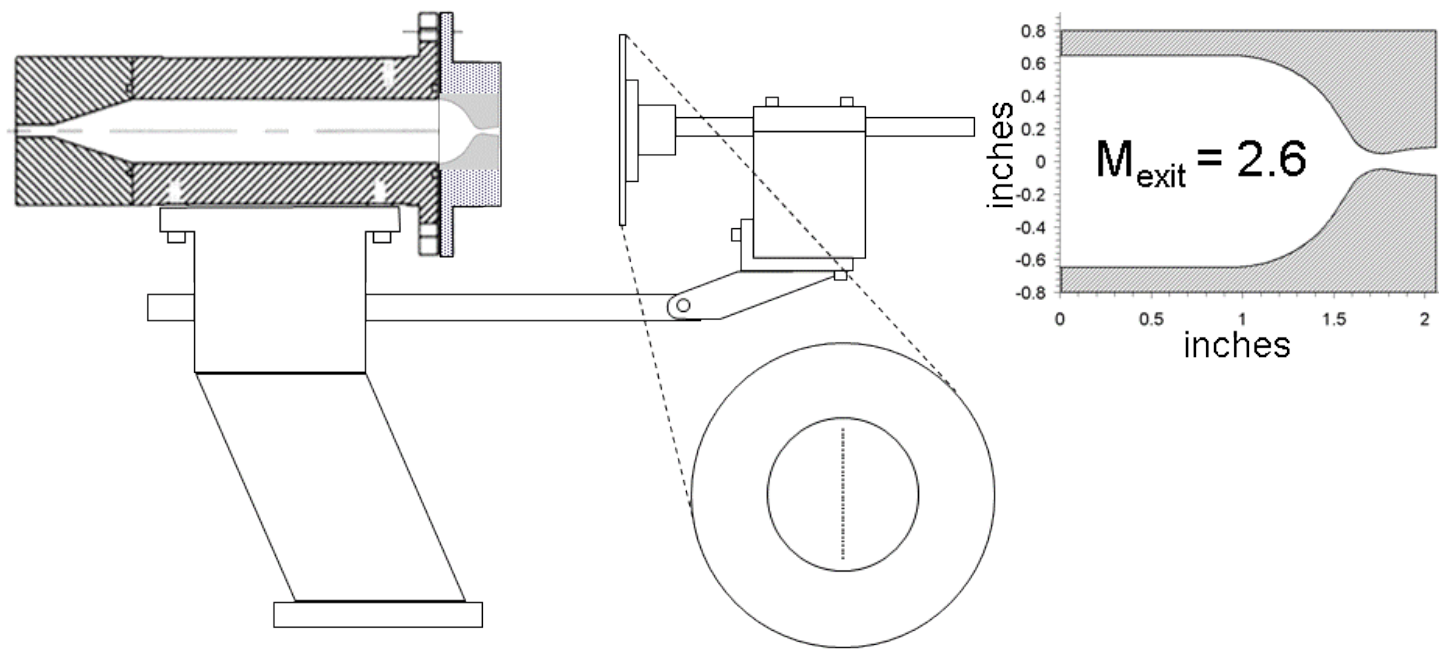

Figure 1. Test Hardware. Schematic of plenum is shown with heat tape and insulation removed. 


\section{B. Model Configuration Parameters}

The full range of test cases is presented in Table 1 in case the interested reader wishes to obtain additional data from the authors at different configurations than those covered in the present paper. The controlling limitation on the ranges of testable Reynolds numbers and pressure ratios was the inability to achieve steady chamber pressures below 1 or 2 Torr $(0.2-0.4$ psi). $)$ Velocity measurements reported in this paper were obtained for two configurations. Both configurations used nitrogen seeded with a mixture of 5\% NO in helium (with resulting NO mole fractions of approximately $0.5 \%$ ). Both configurations used the supersonic nozzle and a $90^{\circ}$ impingement angle. Configuration \#1 had an impingement distance of $44.5 \mathrm{~mm}$ (1.75 in.; 10.7 nozzle diameters), while configuration $\# 2$ had an impingement distance of $63.5 \mathrm{~mm}$ (2.5 in.; 15.2 nozzle diameters).

For each hardware configuration, two flow parameters were varied: the exit Reynolds number $\left(\operatorname{Re}_{\text {exit }}\right)$ and the JPR. $\operatorname{Re}_{\text {exit }}$ was defined in terms of the nozzle exit diameter, $D_{e}(4.17 \mathrm{~mm}, 0.164 \mathrm{in}$. for the supersonic nozzle), and the density $\rho_{e}$, velocity $V_{e}$, and dynamic viscosity $\mu_{e}$ at the nozzle exit, as given by Eq. (1).

$$
\operatorname{Re}_{e x i t}=\frac{\rho_{e} V_{e} D_{e}}{\mu_{e}}
$$

$\mathrm{Re}_{\text {exit }}$ was varied by changing the mass flow rates (using mass flow controllers), which changed the plenum pressure, and by changing the plenum temperature by varying the voltage to heat tape that was wrapped around the plenum hardware. $J P R$ was defined as the ratio of the static pressure at the nozzle exit, $p_{e}$, to the ambient pressure in the test section, $p_{a}$, according to Eq. (2), and was varied by changing the test section pressure for a given Reynolds number (and therefore, a fixed $p_{e}$ ).

$$
J P R=\frac{p_{e}}{p_{a}}
$$

\section{PLIF Flow-Tagging Velocimetry Technique}

The PLIF laser system includes a tunable Nd:YAG-pumped dye laser followed by doubling and mixing crystals. The resulting output, at $226.256 \mathrm{~nm}$, was tuned to excite the strongly fluorescing spectral lines of NO near the band head of the $\mathrm{Q}_{1}$ branch ( $\mathrm{Q}$ denotes a change in rotational quantum number equal to zero). Optics formed the beam into a laser sheet that was $100 \mathrm{~mm}$ wide by $\sim 0.2 \mathrm{~mm}$ thick (FWHM) in the measurement region. The laser sheet entered the test section through an ultraviolet-grade fused silica window approximately 1 meter above the measurement region. Fluorescence was imaged onto a gated, intensified CCD (charge-coupled device; an electronic light sensor, the intensifier of which makes the CCD capable of short exposures - or gates - and single photon detection with sensitivity in the ultraviolet). The viewing angle was normal to the laser sheet. Images with dimensions of 512 pixels x 512 pixels were acquired at $10 \mathrm{~Hz}$ with a $200 \mathrm{~ns}$ camera gate width and a spatial resolution of 6.8 pixels $/ \mathrm{mm}$. This system is detailed in Ref. 3 and Ref. 4.

To obtain velocity measurements, a hair comb was inserted in the laser sheet, creating evenly-spaced shadows in the sheet. The NO molecules absorb this light and then emit photons via the de-excitation process of fluorescence. Since the fluorescence lifetime is 100-200 ns, depending on the operating conditions, the molecules continue to fluoresce as they convect downstream. By shortening the camera gate width $\left(t_{G W}\right)$ to $200 \mathrm{~ns}$ and using a camera delay, $\left(t_{G D}\right)$ of 500,750 , or $1000 \mathrm{~ns}$, images of the displaced NO molecules were obtained. Comparing the delayed image to a reference allows the gas velocity to be determined: the velocity is equal to the distance moved divided by the time delay between laser and camera. However, we observed that using an image with a zero time separation between laser firing and camera acquisition as the reference could occasionally lead to significant (several hundred $\mathrm{m} / \mathrm{s}$ ) systematic errors in the measurement. This error was caused, perhaps, by vibrations and drift of the equipment that occurred between the zero delay and delayed measurements. Consequently an alternate method has been 
developed and presented for the first time in this paper: the velocity far from the jet axis (between about 3.5 and 5.3 nozzle diameters away from the jet centerline) has been assumed to be zero, or close to zero. These data have been used as a reference zero for the measurements in the jet core. Since both signal and reference velocity data are obtained simultaneously, this method eliminates the error inherent to the previous method. However, if the actual velocity in this region is nonzero, it will lead to a direct error in the measured velocity since the technique calculates the relative velocity between the jet flow and the flow in this reference region. This assumption of zero jet velocity far from the jet core was loosely validated using computational fluid dynamics simulations of similar non-impinging jet flows (see section II.D.3 below for more details).

\section{Data Processing Methodology}

\section{Dotcard Unwarping Procedure}

In order to correct for perspective and lens distortion, a calibration image was acquired with a dotcard positioned in the plane of the laser sheet. (A dotcard is simply a two-dimensional rectangular array of square dots of known size and known spacing. In these tests, black square dots placed every 0.25 in. were printed on white card stock, which was then affixed to a rigid plate of corrugated aluminum.) The optical access in these experiments permitted perpendicular viewing of the measurement plane. The perspective and/or lens distortion was found to be relatively minor for all velocimetry runs; however, even these relatively minor distortions were found to lead to velocity errors on the order of $40 \mathrm{~m} / \mathrm{s}$, if not corrected. Correcting for these distortions involves using a customized version of the publically available UnwarpJ plugin for the ImageJ software package available from the National Institutes of Health. Figure 2 illustrates each step of the image registration process.

First, the raw image (Fig. 2(a)) is converted to a black and white image and a black mask is applied to areas outside the plane of the dotcard (Fig. 2(b)). (Unfortunately, the presence of the impingement disk at 10.7 nozzle diameters restricted the size of the dotcard, and time constraints prevented the acquisition of an image of a larger dotcard with the impingement disk at 15.2 nozzle diameters.) Second, a target dotcard with dots of identical size and spacing to the imaged dotcard was generated (Fig. 2(c)). Third, to prevent the unwarping procedure from greatly distorting the right edge of images, thin white registration lines were added to both the black and white image of the dotcard and to the target dotcard image (Fig. 2(b) and 2(c)). Fourth, a transformation mapping the
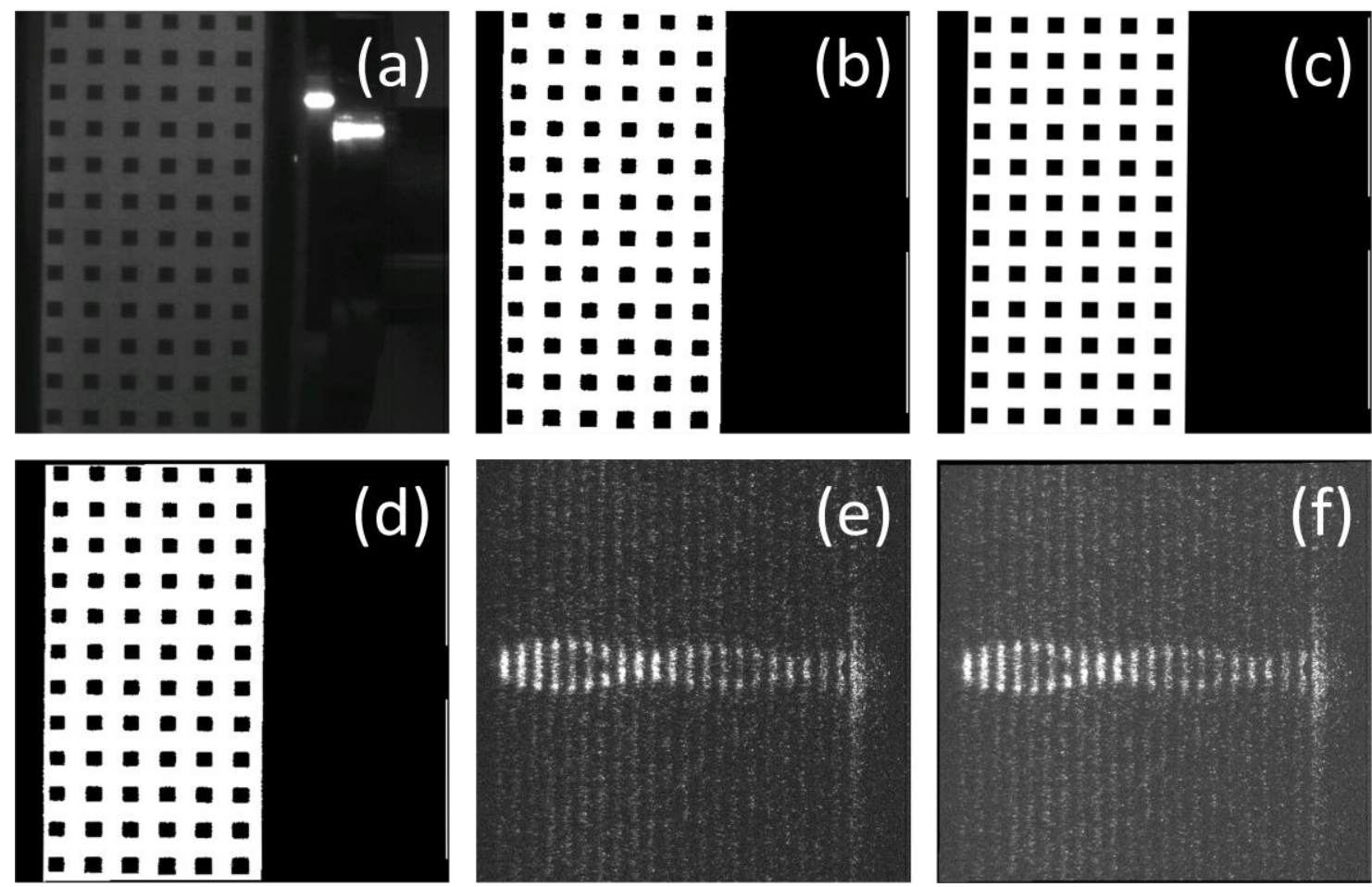

Figure 2. Dotcards and Unwarping Procedure. (a) Raw image of dotcard. Impingement disk hardware is visible on the right of the image. (b) Black and white dotcard, including thin white registration lines. (c) Target dotcard. (d) Registered (aka "unwarped") dotcard. (e) Raw single-shot PLIF image for a case with hardware configuration \#2. (f) Registered single-shot PLIF image. 
black and white dotcard to the target dotcard was calculated using the UnwarpJ plugin for ImageJ. Fifth, this transformation was applied to all experimental PLIF images (which had been converted to tif images from their original .spe format). Figure 2(d) shows the registered dotcard image after the application of this transformation. Figure 2(e) shows a raw, single-shot PLIF image and Fig. 2(f) shows the registered version of this image after the transformation has been applied. A thin black border is visible in the upper left and bottom right corners of Fig. 2(f). The relatively minor difference between the raw and registered images indicate that the camera was positioned very nearly normal to the imaging plane and that the imaging system had very little distortion. In order to prevent loss of data and to preserve the large dynamic range of the original data, the UnwarpJ plugin was modified to save the registered images as 32-bit .tif images, in addition to being extended to operate in batch mode

\section{Determination of Magnification}

In addition to allowing image registration, dotcards also provide a convenient means for determining the magnification (i.e. spatial scale) of experimental images. Figure 3 illustrates two steps in this process. Intensity data are plotted along a line through the registered dotcard image. The discrete derivative of intensity is calculated in order to identify the edges of each dot. The location of either edge is then plotted as a function of the known dot spacing, and the slope of a line fitted to these data yields the magnification in units of pixels $/ \mathrm{mm}$. In practice, this procedure was carried out along several lines of dots, both horizontal and vertical, near the center of the image and along the edges. The average magnification was then found and the variations in the values were used to estimate the uncertainty associated with this calculation. See the section below for details on the contribution of this error to the overall measurement uncertainty analysis.
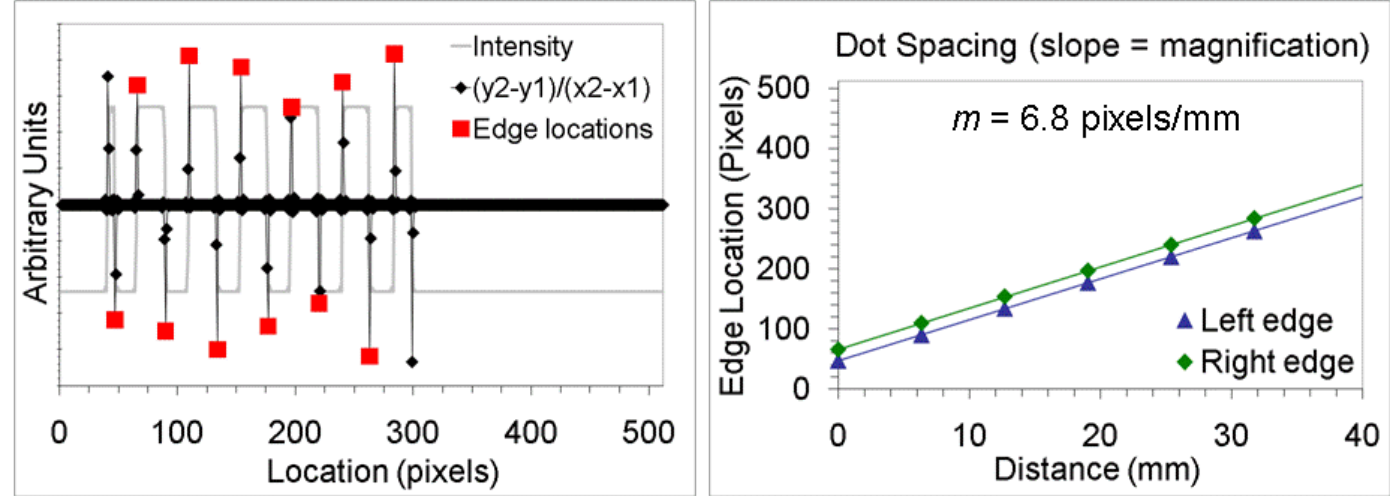

Figure 3. Determining Magnification. (a) Discrete derivative of intensity is used to determine the location of the edge of each dot. (b) The measured location of the dot edges plotted versus known dot spacing yields a magnification of $m=6.8$ pixels $/ \mathrm{mm}$

\section{Determination of Spatial Displacement}

Once the PLIF images have been unwarped, a MATLAB image processing procedure is used to determine the location of the center of each line in each of 100 single-shot images. Figure 4 illustrates the procedure conceptually. An 11 row by 5 column averaging filter is applied to each registered image to remove high-frequency noise (this step is not shown in Fig. 4). The smoothed images are then processed one row at a time. Each row is divided into sections along the approximate average location of the shadows between each bright fluorescent line. A second order polynomial is then fit to the brightest 9 pixels within each section. The center of this fitted parabola is then recorded as the measured line location for that measurement location. (Measurement locations exist at each row within each line, where Line 1 is the leftmost fluorescent line.) Measurements are rejected if the fitted parabola is found to either have a line center outside the physical extent of the measurement location or if it is concave-up. Since the algorithm is supposed to be fitting to the center of a bright line, the fitted parabola should be concavedown. A concave-up fit is either a fit to noise or possibly a fit to the center of the shadow between lines. Because 100 single-shot images are processed in this manner for each run, up to 100 independent measurements of the line center are made at each measurement point (the number of independent measurements can be less than 100 if any of the fits were rejected). An average line location is then calculated and exported to a spreadsheet.

Note that we are not entirely satisfied with the present algorithms for identifying bad fits. In locations with poor signal-to-noise ratios, they may introduce bias errors that favor the center of the measurement section. (To see why this is the case, imagine fitting to purely random data. Fits which gave line locations outside the bounds of each section would be rejected, while those not rejected would be randomly distributed within the measurement section, leading to an average line location at the center of the section). Improving these algorithms will be a primary goal of future work. 

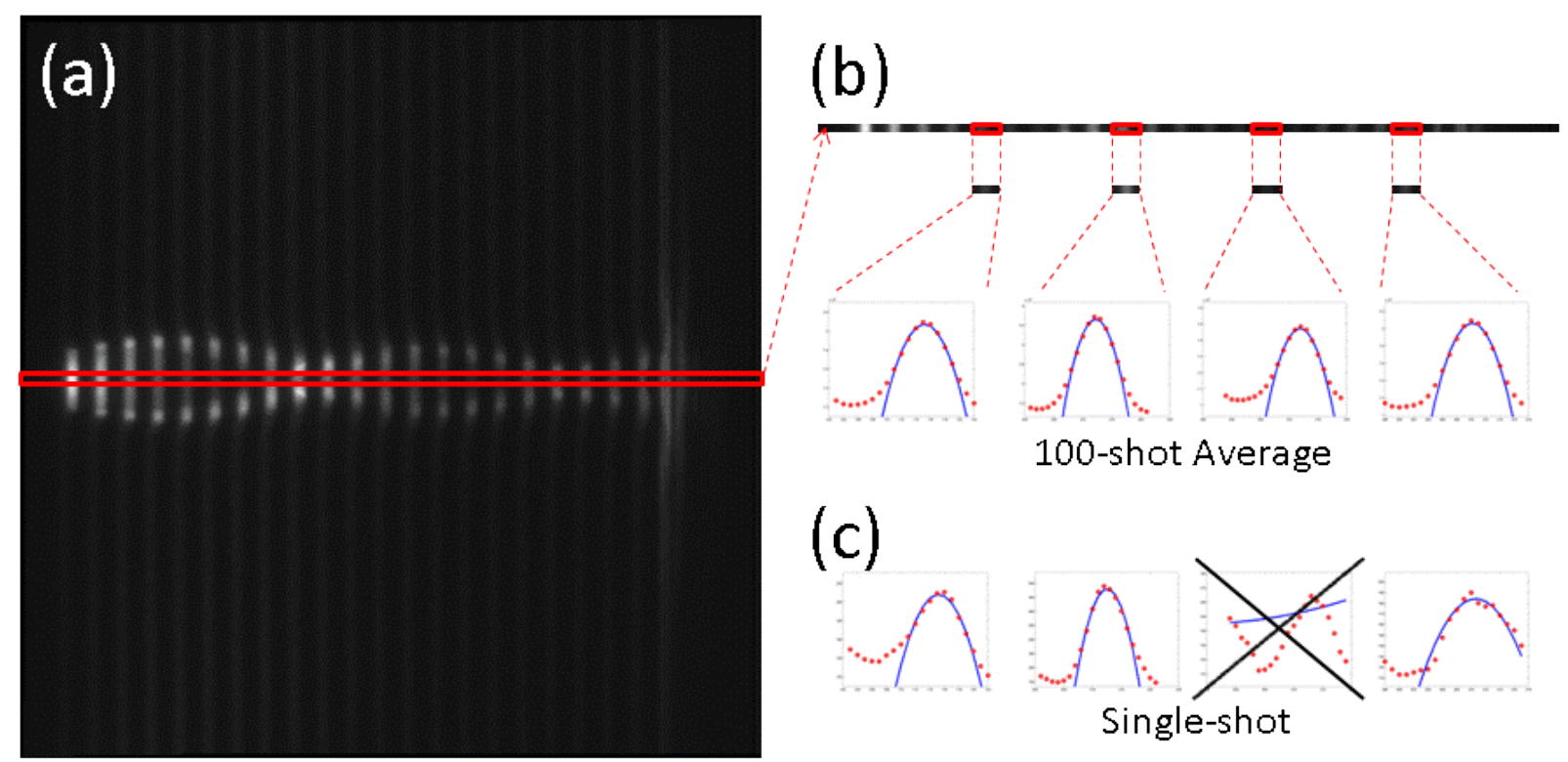

Figure 4. Data processing procedure. (a) Registered 100-shot average PLIF image (b) Selected (centerline) row, showing intensity data with fitted parabolas for $5^{\text {th }}, 10^{\text {th }}, 15^{\text {th }}$, and $20^{\text {th }}$ lines for a 100 -shot average image for a case with hardware configuration \#2. (c) Intensity data and fits for a single-shot image, including a rejected poor fit.

Once average line locations have been recorded for each measurement point, velocity is calculated through a series of spreadsheets. First, a zero-velocity reference line must be determined. These reference lines correspond approximately to the geometric center of the open slots in the hair comb. However, reference lines must be measured using the lines of fluorescence because if the laser sheet is not perfectly collimated, the reference lines will not be exactly parallel to one another. Reference regions are selected that are presumed to be adequately far from the jet so as to have a velocity sufficiently close to zero while avoiding the very edges of the image where distortions due to the registration process tend to be greatest.

CFD solutions for three laminar free (non-impinging) jet cases with a sonic nozzle indicate velocities of less than $5 \mathrm{~m} / \mathrm{s}$ in regions at least 1 jet diameter away from the core jet flow. (The velocity results from these CFD solutions are, as yet, unpublished. However, other parameters from these solutions, including temperature, pressure, and mixing fraction, were used to compute theoretical PLIF images. Those theoretical PLIF images and details about the input parameters used to generate these CFD solutions can be found in Ref. 4.) Experimental digital particle imaging velocimetry (DPIV) measurements of an impinging jet issuing from a sonic nozzle have been reported by Henderson et al. ${ }^{9}$ Their jets issued into atmospheric pressure and were at a higher, turbulent Reynolds number than any of the runs in this paper. Their nozzle had an exit diameter of $25.4 \mathrm{~mm}$, an exit Mach number of 1, and was housed in a $30^{\circ}$ cone, as opposed to our nozzle, which had a sonic throat diameter of $2.4 \mathrm{~mm}$, an exit diameter of 4.2 $\mathrm{mm}$, an exit Mach number of 2.6 and issued through a plate normal to the nozzle exit. Their most similar test condition was for a jet with a $J P R$ of 2.1 (nozzle pressure ratio $p_{d} / p_{a}$ of 4.06) and an impingement distance of $D_{\text {imp }} / D_{e}=4.16$. Computations of this same flow field using large eddy simulation (LES) have been performed by Dauptain et al. ${ }^{10}$ Both experimental and computational results have colormaps with limited resolution at the low end of the velocity scale, but both indicate velocities consistent with the minimum value on their respective scales in the nearfield region just outside the jet (radially, about one and a half nozzle diameters away from the jet centerline). For the computational (LES) results, the velocity in this region was less than $50 \mathrm{~m} / \mathrm{s}$ and for the experimental (DPIV) results, it was less than $20 \mathrm{~m} / \mathrm{s}$.

For most runs, the jet boundaries were defined to be approximately \pm 1.5 jet diameters away from the jet centerline, hopefully giving a conservative definition of the region where flow velocity was assumed to be negligible. (A jet diameter is defined to be the approximate diameter of the fully expanded jet plume.) A reference line was calculated using the 50 rows above the upper boundary of the jet and the 50 rows below the lower boundary of the jet. A least-squares fit to the measured line locations in these reference rows yielded a slope and intercept for each reference line. Data from these reference locations are shown for a sample run as thick lines in Fig. 5 while fitted reference lines are shown as thinner lines. At each measurement location, the difference in the measured line 
location and the fitted reference line is converted to a physical displacement $\Delta x$ by dividing by the magnification, $m$ (6.82 pixels $/ \mathrm{mm})$.

In theory, each registered image could be processed individually to determine velocity on a single-shot basis. In practice we found the signal intensity in the reference region to be too low to do so and as a result, the results presented herein are time-averaged velocities. An intermediate approach is suggested for future work. The reference lines would be computed from the average of the individual measurements, as was done in the current work. Measured line locations within the jet (where the signal is higher) from one single-shot image could then be compared with these reference lines to obtain a measurement of instantaneous rather than time-averaged velocity.

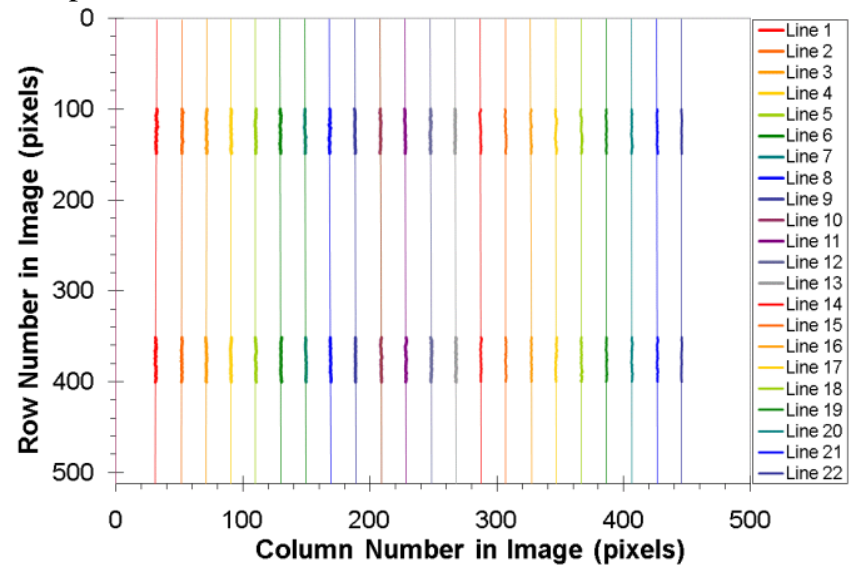

Figure 5. Zero-velocity reference lines for the case in Figs. 2 and 4. Measured line locations are shown as thicker lines while fitted reference lines are shown as thinner lines image for a case with hardware configuration \#2. Run 506.

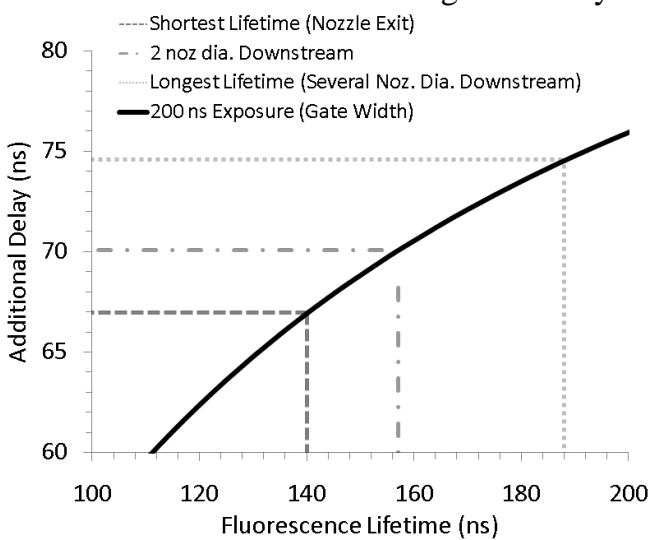

Figure 6. Additional delay, $t_{\text {add }}$. Solid line indicates calculated values resulting from Eqs. 7 and 8. Dashed lines indicate the shortest and longest lifetimes expected for the range of conditions encountered in these tests and the corresponding $t_{\text {add }}$.

\section{Determination of Temporal Delay}

In order to calculate velocity from this measured displacement, the effective delay after the laser pulse, $\Delta t$, is needed. The effective delay time is that time at which half of the total fluorescence would have been acquired. By modeling the fluorescence intensity as an exponential decay and integrating the intensity from the time the intensifier gate opens, $t_{G D}$, to the time it closes, $t_{G D}+t_{G W}$, we arrive at Eq. (3) where $I_{\text {total }}$ is the total acquired intensity in arbitrary units.

$$
I_{\text {total }}=\int_{t_{G D}}^{t_{G D}+t_{G W}} e^{-t / \tau_{L I F}} d t
$$

After integration, Eq. (3) becomes

$$
I_{\text {total }}=-\tau_{L I F}\left[e^{-\left(t_{G D}+t_{G W}\right) / \tau_{L I F}}-e^{-t_{G D} / \tau_{L I F}}\right]
$$

If the fluorescence lifetime $\tau_{L I F}$ were infinite - that is, if fluorescence intensity did not decay with time, or was at least effectively constant during the exposure time (i.e. intensifier gate width, $t_{G W}=200 \mathrm{~ns}$ ) - then $\Delta t$ would simply be the gate delay $t_{G D}$ plus half the gate width, $t_{G W}$. However, because $\tau_{L I F}$ is on the order of the exposure time, significantly more fluorescence is acquired near the beginning of the exposure than at the end. As a result, the effective time delay is less than $\left(t_{G D}+1 / 2 t_{G W}\right)$. In order to determine the time at which half the total intensity has been acquired, a second expression is needed. Equation (5) states that half the total intensity will be acquired between the time the intensifier gate opens, $t_{G D}$, and some as-yet-undetermined time $\Delta t$.

$$
\frac{1}{2} I_{t o t a l}=\int_{t_{G D}}^{\Delta t} e^{-t / \tau_{L I F}} d t
$$

After integration, Eq. (5) becomes

$$
\frac{1}{2} I_{\text {total }}=-\tau_{L I F}\left[e^{-\Delta t / \tau_{L I F}}-e^{-t_{G D} / \tau_{L I F}}\right]
$$


By substituting the right hand side of Eq. (4) for $I_{\text {total }}$ and solving for $\Delta t$, we arrive at the following expression:

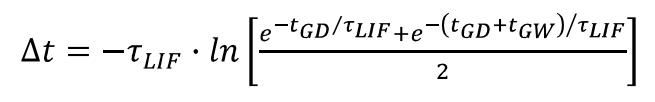

Since $\Delta t$ is a function of the specific gate delay chosen, we have elected to calculate the portion of $\Delta t$ that is solely a function of the fluorescence lifetime $\tau_{\mathrm{LIF}}$. This quantity has been named the additional delay, is labeled $t_{\text {add }}$, and is related to $\Delta t$ by Eq. (8).

$$
\Delta t=t_{G D}+t_{a d d}
$$

Figure 6 shows $t_{\text {add }}$ as a function of $\tau_{\mathrm{LIF}}$. We estimated the range of fluorescence lifetimes expected for the present test conditions. Short lifetimes exist when quenching is highest, which occurs at high pressure (e.g. near the nozzle exit and for a high Reynolds number case) and/or low temperature (e.g. in the most expanded part of the jet for a high $J P R$ case). By contrast, low quenching occurs at low pressures (e.g. relatively far downstream where the jet has fully expanded for a case with low ambient pressure) and/or high temperatures. Using measured plenum and test chamber pressures and temperatures along with isentropic compressible flow equations, fluorescence lifetimes of between $140 \mathrm{~ns}$ and $188 \mathrm{~ns}$ are expected for the range of conditions encountered in these tests. However, for all but the first (leftmost) line, this range is $157 \mathrm{~ns}$ to $188 \mathrm{~ns}$, which gives an additional delay of approximately $72.5+/-$ $2.5 \mathrm{~ns}$. For the first line, we increased the uncertainty in the additional delay, $t_{\text {add }}$, to $+2.5,-5.5 \mathrm{~ns}$, in order to account for the wider range of possible fluorescence lifetimes in this portion of the flow. The runs presented in this paper used a gate delay of $t_{G D}=750 \mathrm{~ns}$, resulting in a $\Delta t$ of $822.5 \mathrm{~ns}$.

5. Error Analysis

The overall calculated uncertainty in the velocity measurement at a given measurement location, $i$, is given by the following expression:

$$
u_{v_{i}}=\sqrt{\left(\frac{u_{r e f}}{\Delta t}\right)^{2}+\left(\frac{\sigma_{\text {line loc }}}{\Delta t \cdot \sqrt{n_{\text {valid }}}} c_{95 \%}\right)^{2}+\left(\frac{u_{t}}{\Delta t} v_{i}\right)^{2}}+\frac{u_{m a g}}{m} v_{i}
$$

Computed values for $u_{v}$ within the jet flow (as opposed to in the nearly-stagnant region far from the jet flow) were typically between 15 and $50 \mathrm{~m} / \mathrm{s}$. Here, $u_{r e f}$ is the uncertainty (in meters) in the location of the zero-velocity reference point; $\Delta t$ is the effective time delay, given in Eq. (4); $\sigma_{\text {line loc }}$ is the standard deviation in the measured line location at a given measurement location; $n_{\text {valid }}$ is the number of valid measurements made at a given measurement location (this number is typically 100 in the jet and less than 100 outside the jet where the signal to noise ratio is lower); $c_{95 \%}$ is the $95 \%$ confidence interval factor of $1.96 ; u_{t}$ is the total timing uncertainty (calculated using Eq. (11) below); $u_{m a g}$ is the uncertainty in the magnification, $m$ (for locations downstream of the region covered by the dotcard used in image registration, this factor has been increased by an additional 10\%); and. $v_{i}$ is the measured velocity at a given measurement location, given below in Eq. (10).

$$
v_{i}=\frac{\Delta x_{i}}{\Delta t}(1+A)
$$

The additional term, $A$, is a correction factor related to the finite fluorescence lifetime, the finite intensifier gate width, and the fact that fluorescing molecules with a non-zero velocity move during the exposure time. Without this correction term, an error proportional to the velocity would be introduced since more fluorescence intensity is acquired during the beginning of the exposure than during the end of the exposure. For a positive velocity, this means that the imaged line of molecules will be brighter on the left side of the line than on the right side. See Ref. 11 for an in depth description of this effect and expressions for calculating this correction factor for different gas compositions, fluorescence lifetimes, and gate widths.

One potentially large source of error has been neglected in the preceding analysis. This error arises in regions where there is a left-to-right gradient in the amount of quenching across the width of a measurement line. Quenching is primarily a function of pressure. Therefore, if strong pressure gradients exist between the left and right edges of a measurement line, significant errors could result. The two places in underexpanded jet flows where 
this is likely to occur are near the nozzle exit (that is, along the first measurement line within the jet) and across normal shock waves. The quenching gradient near the nozzle exit would reduce the signal level on the left side of the line (higher pressure leads to higher quenching) more than on the right side of the line. This would lead to a right-shifted line center and, consequently, an artificially high velocity measurement. We estimate this error to be as large as $275 \mathrm{~m} / \mathrm{s}$ for some cases. Therefore, all velocity measurements along Line 1 should be treated as suspect, or at best, used as an upper bound on the velocity at that point. The alignment of flow features like a Mach disk (normal shock wave) with the laser comb happens by chance, if at all, and so the associated error of making a measurement along a line straddling a Mach disk affects only a few cases. The quenching gradient across a shock wave (low quenching upstream, high quenching immediately downstream) could potentially lead to a line which is brighter on the left. However, other competing effects, like the increase in density across a shock, may lead to almost constant signal intensity across the shock, reducing the effect of this error. Suffice it to say that caution should be exercised in interpreting measurements along a normal shock wave, if for no other reason than that strong velocity gradients exist within such a measurement volume.

The total timing uncertainty $u_{t}$ is given by

$$
u_{t}=\sqrt{u_{j i t t e r}^{2}+u_{G D}^{2}+u_{\Delta t}^{2}}
$$

where $u_{\text {jitter }}$ is the timing jitter of the laser relative to the trigger signal; $u_{G D}$ is the gate delay uncertainty (i.e. the timing of the opening of the intensifier gate relative to the laser pulse); and $u_{\Delta t}$ is the uncertainty in the effective time delay due to the finite fluorescence lifetime and non-instantaneous exposure time.

Table 2 contains values of many of these factors for the present experiments. Note that a negative timing uncertainty adds to the positive error bar in velocity uncertainty, whereas a positive timing uncertainty contributes to a negative error bar in velocity uncertainty because shorter delay times mean higher computed velocities and vice versa. The largest contribution to the overall uncertainty is, by far, the uncertainty in the measured line locations. This uncertainty can be reduced by increasing the number of measurements (i.e. the number of single-shot images), improving the signal-to-noise ratio in the images, and/or increasing the magnification of the imaging system.

\begin{tabular}{l|ll}
\hline \multicolumn{2}{|c|}{ Constants and Values for Uncertainty Analysis } \\
\hline$\Delta t$ & Value (for constants) & Typical Contribution to Uncertainty \\
$\tau_{\mathrm{LIF}}$ & $222.5 \mathrm{~ns}$ & \\
$u_{\text {jitter }}$ & $\pm 1 \mathrm{~ns}$ & $\pm 0.1 \%$ \\
$u_{G D}$ & $\pm 3 \mathrm{~ns}$ & $\pm 0.4 \%$ \\
$u_{\Delta t}$ & $\pm 2.5 \mathrm{~ns}(+2.5,-5.5 \mathrm{~ns}$ for Line 1$)$ & $\pm 0.7 \%$ \\
$u_{t}$ & $\pm 4.0 \mathrm{~ns}(+4.0 \mathrm{~ns},-6.3 \mathrm{~ns}$ for Line 1$)$ & $\pm 0.5 \%(+0.8 \%,-0.5 \%$ for Line 1$)$ \\
$u_{\text {ref }}$ & variable for each line & $\pm 10 \mathrm{~m} / \mathrm{s}($ typical value) \\
$\sigma_{\text {line loc }}$ & variable for each measurement point & $\pm 150 \mathrm{~m} / \mathrm{s}$ (typical value) \\
$\sqrt{n_{\text {valid }}}$ & & \\
$n_{\text {valid }}$ & typically $60-100$ outside jet, 100 inside jet \\
$u_{\text {mag, }, 1}$ & \pm 31.90 pixels $/ \mathrm{m}$ & $\pm 0.47 \%$ \\
$u_{\text {mag, }, 2}$ & \pm 35.09 pixels $/ \mathrm{m}$ & $\pm 0.51 \%$ \\
$m$ & 6,818 pixels $/ \mathrm{m}$ & \\
$c_{95 \%}$ & 1.96 & \\
$A$ & 0.0257 & \\
\hline
\end{tabular}

Table 2. Uncertainty parameters and resulting contributions to overall uncertainty.

\section{Experimental Results}

Table 1 lists the number of cases that were studied for each combination of hardware configuration and type of PLIF imaging, including velocimetry, that were investigated in these tests. Reynolds numbers and jet pressure ratios were calculated based on nozzle exit conditions, as defined in Eqs. (1) and (2). In the current paper, only a subset of the impinging jet velocimetry cases is analyzed, namely those cases with a zero-velocity flow region containing enough NO to allow a high-quality zero velocity reference. The presence of the impingement plate traps NO in the region around the jet, whereas in free-jet cases, the jet expands into a large vacuum chamber and there is no signal 
far from the jet to use as a reference. Consequently, none of the free-jet cases had a suitable zero velocity region outside the jet and therefore shall not be analyzed in this paper.

\section{A. Velocity Profiles}

A raw velocimetry image of an impinging underexpanded supersonic jet was shown in Fig. 2(e). The flow is from left to right. The jet is impinging on a flat surface located about $7 / 8$ of the way down the image. The laser sheet is passing from top to bottom. The comb has been placed in the laser sheet above the flow, creating the shadows observed as vertical stripes in the flow. Note that this resulted in 15 measurement lines for the cases with the smaller impingement distance of $D_{\text {imp }}=10.7$ nozzle diameters, and 22 lines for cases with the larger impingement distance of $D_{i m p}=15.2$ nozzle diameters. Prior analysis of full PLIF images obtained for this case indicated that the flow is laminar with a small degree of unsteadiness. ${ }^{5}$ The image in Fig. 2(e) is a single-shot image obtained during a $200 \mathrm{~ns}$ exposure beginning $750 \mathrm{~ns}$ after the laser excitation, so the fluorescence patterns are not straight lines. (Images acquired at 0 ns delay show straight vertical lines in the images). Far from the center of the jet, the fluorescence lines are observed to be straight. The velocity in this region, far from the jet core, is assumed to be zero for the purposes of measuring the velocity in the jet. One hundred such images were processed to determine the center of each line (as described in section II.D.3. above), and the results combined to determine the average line location at each measurement point. Lines were then fit to data in regions assumed to have negligible velocity relative to the jet flow. Fifty rows above and fifty rows below the jet were used to determine these reference lines.

Figure 7 shows velocity profiles resulting from the processing of this data. The difference between thin (reference) and thick (data) lines is proportional to the flow velocity. The length of the grey lines indicate the velocity scale (longest to shortest: $1000,800,600,400,200,100,50 \mathrm{~m} / \mathrm{s}$ ). The gas velocity goes from zero at the edges (by definition) to roughly $1000 \mathrm{~m} / \mathrm{s}$ in the fastest part in the core of the jet. For comparison, a simple isentropic expansion from the nozzle reservoir conditions to an infinite Mach number (all of the thermal energy being converted kinetic energy) yields velocities of 1,008 to 1,080 meters per second for the range of plenum temperatures encountered in these tests.

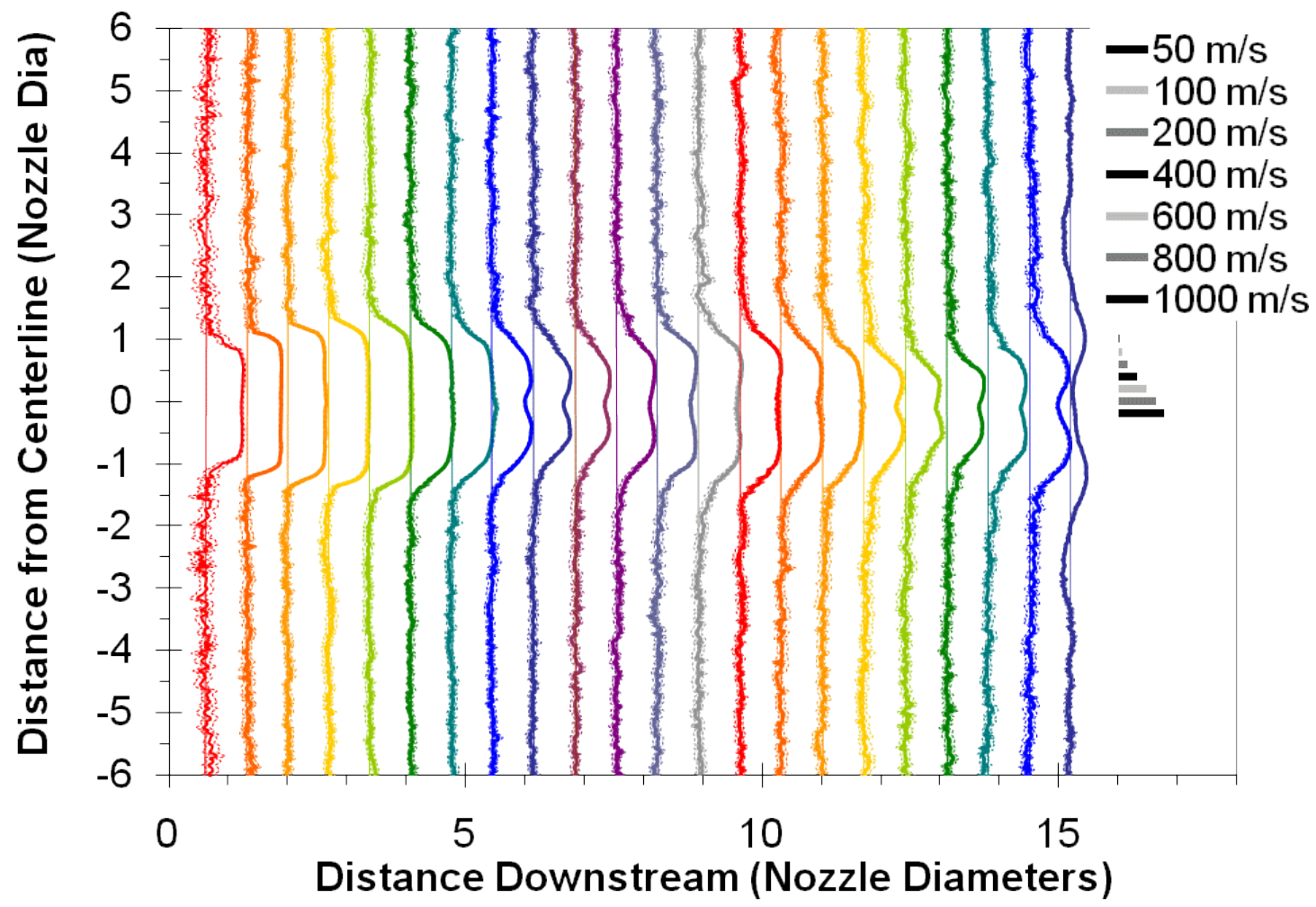

Figure 7. 22 Velocity Profiles with Error "Bars" and Zero-Velocity Reference Lines for the case shown in Figs. 2, 4, and 5. Lines are numbered sequentially, beginning with Line 1 on the left and ending with Line 22 on the right. Dashed lines show the upper and lower $95 \%$ confidence intervals. Hardware configuration $\# 2\left(D_{\text {imp }}=\right.$ 15.2 nozzle diameters). Run 506. 

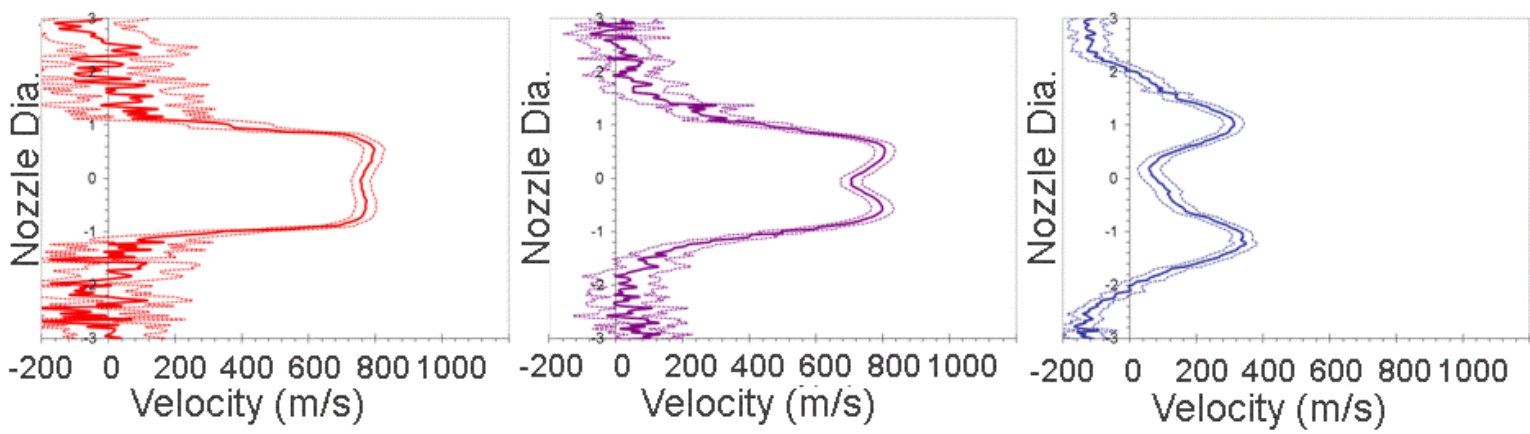

Figure 8. Velocity Profiles with Error "Bars" for Lines 1, 11, and 22 from Fig. 7. In Fig. 7, Line 1 is on the far left, Line 22 is on the far right, and Line 11 is in the approximate middle. Run 506.

The velocity profiles initially have a top hat shape, transitioning to a more Gaussian profile, and then developing two peaks downstream as the shear layer on the edge of the jet thickens. Figure 8 shows velocity profiles for three selected lines (Line 1, the red line nearest to the nozzle; Line 11, the purple line approximately halfway between the nozzle and impingement target; and Line 22, the grey-blue line nearest to the impingement target), along with upper and lower $95 \%$ confidence interval bounds, for this same case. Notice the larger bounds (error bars) on the first profile, due to the presumably shorter fluorescence lifetime in the high-pressure (and therefore increased quenching) environment near the nozzle exit, and in regions with poor signal-to-noise ratios. The double-peaked shape of the velocity profile for Line 11 is a result of flow near the edges of the jet which has passed through oblique shocks having a higher velocity than flow near the centerline which has passed through an essentially normal (perpendicular) shock. Nearly stagnated flow near the impingement surface is evidenced by the small velocity seen in the center of the profile for Line 22.

\section{B. Centerline Velocity Measurements}

Velocity measurements along the jet centerline are shown in Figs. 9, 10, and 11. The thumbnail images are cropped 100-shot average PLIF images. The velocity in Fig. 9 is observed to accelerate in the first barrel shock, then to slow down after the oblique shock waves intersect about 5 nozzle diameters downstream. It then accelerates again inside the second barrel shock and the pattern begins to repeat until the centerline flow nears the impingement target (located at $D_{i m p}=15.2$ nozzle diameters), when the velocity drops to nearly zero. Figure 10 is for a similar $J P R$ and $\mathrm{Re}_{\text {exit }}$ but with a smaller impingement distance of $D_{i m p}=10.7$ nozzle diameters. Note the apparent presence of a recirculation shock and small recirculation region near the impingement target, indicated by a sharp drop in velocity followed by a slightly negative (upstream) velocity. Also notice the influence of the increased proximity of the impingement target on the velocity inside the beginning of the second barrel shock (from about 6 to 8 nozzle diameters), indicated by a lower centerline velocity as compared to the case in Fig. 9. As a third example, Fig. 11 shows the measured centerline velocity for a case with the same smaller impingement distance as in Fig. 10, but with a larger JPR. The initial acceleration accompanying the expansion inside the barrel shock is followed by a sharp drop in velocity across the Mach disk (normal shock wave). A large recirculation region is then indicated by the upstream flow (negative velocity) between the Mach disk and the impingement target.

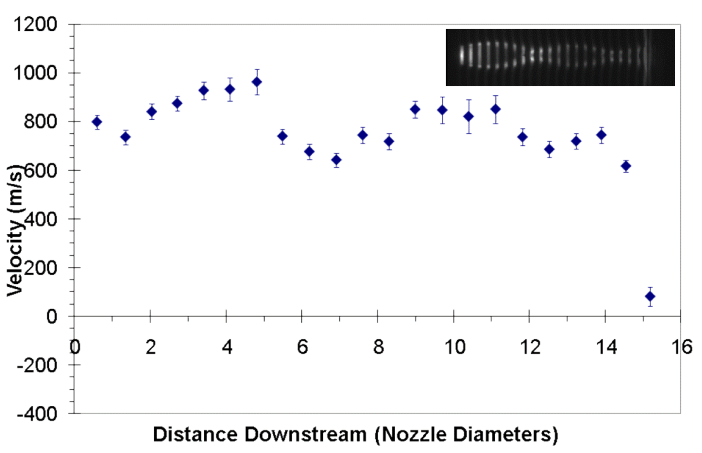

Figure 9. Centerline Velocity. This is the same case as in Figs. 2, 4, 5 and 7. Error bars mark the upper and lower $95 \%$ confidence intervals. Run506.

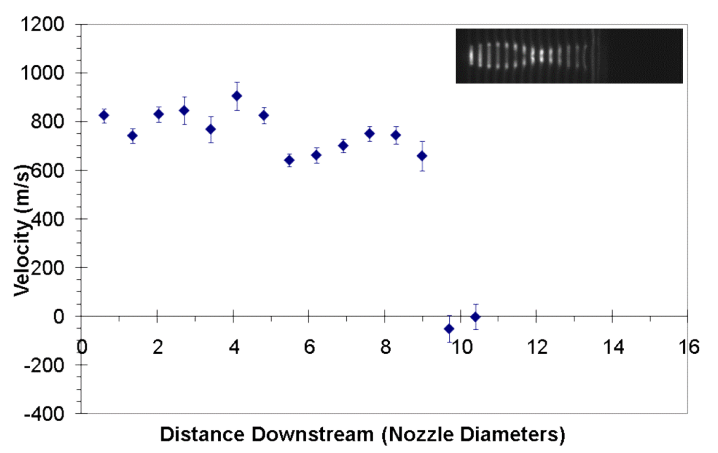

Figure 10. Centerline Velocity. This case has similar conditions as the case in Fig. 8 but with $\mathrm{D}_{\text {imp }}=$ 10.7 nozzle diameters. Run 423. 


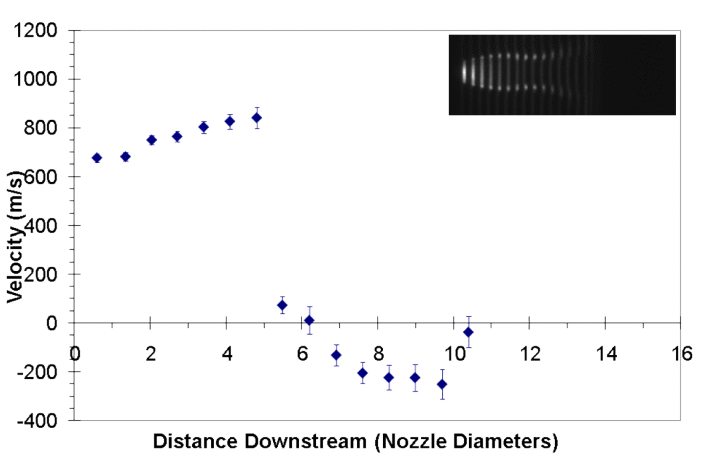

Figure 11. Centerline Velocity showing large recirculation region. This case has the same impingement distance as the case shown in Fig. 10 but with a larger jet pressure ratio. Run425.

\section{Comparison with Impingement Surface Pressure Measurements}

Velocity profiles were found to be instructive in understanding surface pressure measurements, acquired simultaneously with the PLIF images. Due to the small diameter and long length of the tubing connecting the pressure taps to the pressure gauges, these pressure measurements should be regarded as time-averaged rather than instantaneous measurements. Figure 12 shows two velocity profiles - from the measurement line nearest the surface, Line 15, as well as the line just upstream of it, Line 14-and an impingement surface pressure profile for three separate cases at the smaller impingement distance, $D_{i m p}=$ 10.7 nozzle diameters. Note that the pressure measurements have been normalized by the ambient pressure, $p_{a}$.

In Fig. 12(a), the pressure profile shows a double-peaked structure which mimics the structure of the velocity profiles;
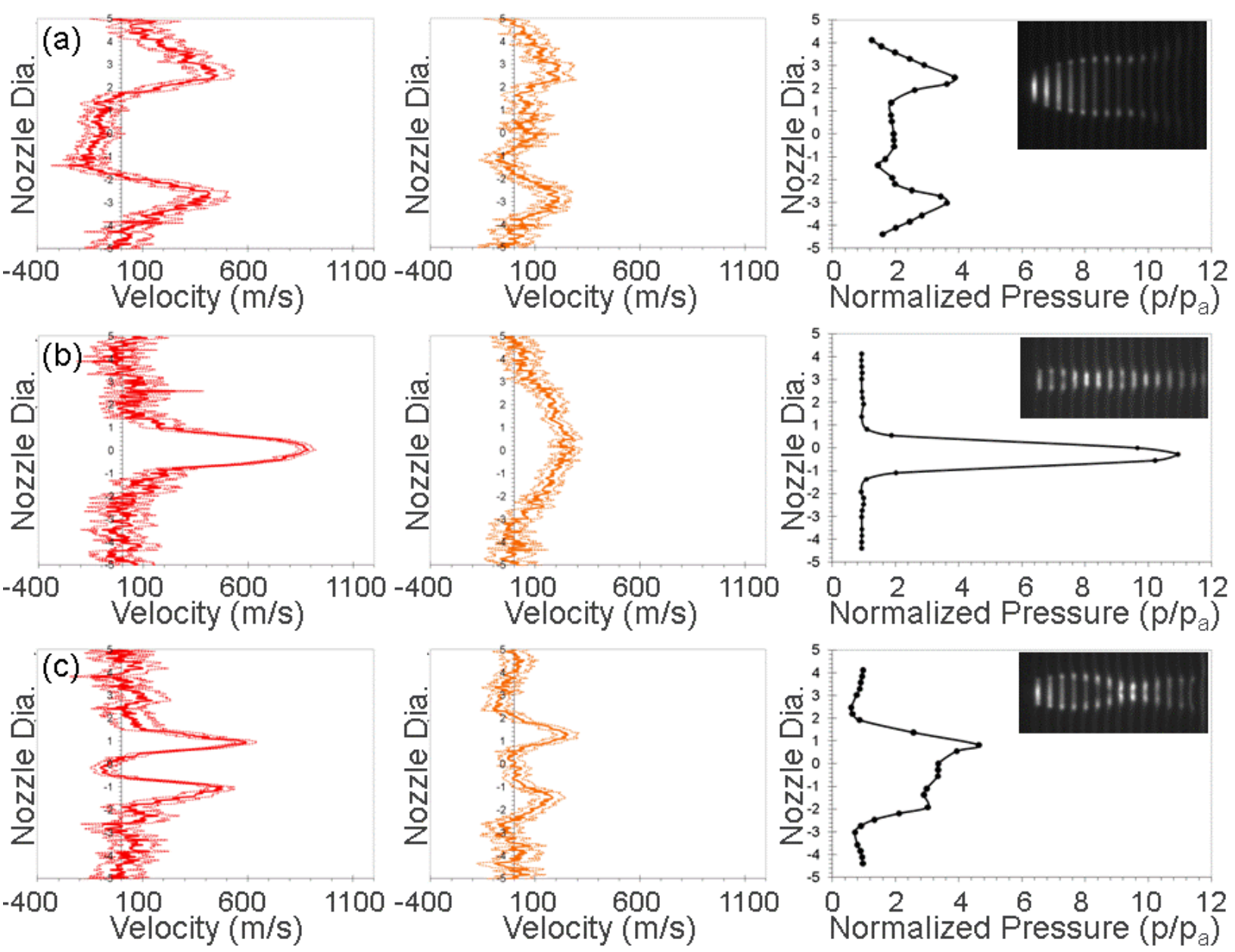

Figure 12. Comparing Velocity and Pressure Profiles. Velocity Lines 14 (red, left) and 15 (orange, center), and impingement surface pressure normalized by ambient pressure (right) with cropped thumbnail 100 -shot average registered images. All three cases are for hardware configuration \#1 and all three thumbnail images are 100-shot average PLIF images. (a) Run 419 (b) Run 429 (c) Run423 
high velocities coincide with high pressures. Figure 12(b) shows a single-peaked profile, both in pressure and velocity. Interestingly, the pressure profile more closely corresponds to the shape of the penultimate velocity profile; the profile just above the surface shows a flattened shape, indicative of the streamwise component of velocity approaching its boundary condition of zero velocity at the impingement surface. In Fig. 12(c), a case is shown with a slight asymmetry in both the pressure and velocity profiles; the upper peak shows a slightly higher pressure and velocity than the lower peak. This asymmetry is not immediately obvious in PLIF images of this case. However, flow visualization images do seem to suggest the presence of a recirculation bubble, which may imply that the flow is near a staging transition (see Ref. 5 for a discussion of recirculation bubbles and staging transitions). This sensitivity near a staging transition may explain why even an infinitesimal non-normal angle in the impingement target would lead to measureable asymmetry in the flow conditions near the surface. Together, this case represents a good example of the synergistic value of simultaneous flow visualization, surface pressure measurements, and velocity measurements; each measurement helps to explain potentially perplexing features of the other.

\section{Suggestions for Future Work and Improvements to the Technique}

A number of suggestions for future work can be made for future applications of this method of flow-tagging velocimetry. First, since the largest contribution to the overall uncertainty of these measurements is the uncertainty in the measured line locations, increasing the number of single-shot images per measurement and/or increasing the magnification of the imaging system would be advisable. In addition, the algorithm for identifying poor fits to the data should be improved and the effects of quenching gradients in the flow should be included in the uncertainty analysis. Decreasing the gate delay would have improved the measurement precision. A gate delay of between 300 and $500 \mathrm{~ns}$ is recommended as a way to increase the average image intensity, especially if the magnification of the imaging system can be increased so that sufficiently large pixel shifts are still observed. Second, modifications to the test apparatus and testing procedure could be made with the intention of more effectively trapping $\mathrm{NO}$ in the zero-velocity region of the flow. Any such modification which helped to increase the signal-to-noise ratio throughout the image (but especially in regions far from the jet) would help to reduce measurement error. For example, an F/2 lens compared to the F/4.5 lens used in this current work would have collected four times more LIF signal. ${ }^{11}$ The use of a custom long-pass filter (recently purchased by our group from Layertec, $\mathrm{GmbH}$ ) in front of the camera lens in place of the UG-5 filter used in these tests would also allow additional fluorescence signal to be collected while still blocking scattered laser light. Third, acquiring a dotcard image which spans the full field of view would help improve the image registration process. It would also help to reduce errors associated with uncertainties in image magnification. Fourth, measurements should be made or CFD performed for impinging jet flows at the conditions of the present tests to ascertain the validity of the assumption of negligible velocity in the reference regions. Finally, Doppler velocimetry could be used to measure the spanwise component of velocity and thus extend the current methodology to a two-dimensional velocimetry technique.

\section{Concluding Remarks}

Quantitative measurements of velocity have been demonstrated using NO PLIF flow-tagging velocimetry. Measured velocities ranged from approximately -200 to $+1,000 \mathrm{~m} / \mathrm{s}$. Accuracies on the order of 15 to $50 \mathrm{~m} / \mathrm{s}$ have been estimated within the jet flows under consideration, with the higher accuracies in regions of higher signal-tonoise. These accuracies, however, rely upon the assumption of zero average velocity in the region between approximately 3.5 and 5.3 nozzle diameters away from the jet centerline. Any deviation from zero average velocity in the reference region will lead to a direct error of the same amount in the measured velocity. Comparisons with pressure measurements along the surface of the impingement target have been made, with the measured velocity profiles serving to explain the major features of these pressure measurements. This velocimetry technique has been shown to have the additional advantage of providing partial flow-visualization since there are a large number of relatively closely-spaced lines through the flowfield. Flow visualization can further the understanding of features in velocity profiles by, for example, showing the location and shape of shock waves and expansion regions. A large dataset suitable for comparison with CFD has been generated for a Mach 2.6 nozzle flow over a range of Reynolds numbers and jet pressure ratios, for two impingement distances, which is planned for publication later this year as a NASA technical memorandum. The single-image technique reported herein theoretically eliminates the limitation of similar two-image techniques which restricts them to measurements in steady flows (or to averaged measurements, with potential systematic biases, in unsteady flows). However, for reliable single-shot measurements of velocity fluctuations in unsteady flows, higher signal-to-noise images are needed. 


\section{Appendix}

Table A1 lists coordinates for the contour of the supersonic nozzle. Table A2 contains parameters for 30 runs20 runs with configuration \#1 and 10 runs with configuration \#2. Run numbers are not consecutive because every other run was acquired with a 0 ns gate delay. (These runs were not used in the present paper but were obtained for use as reference images when implementing an alternative data processing technique requiring separate reference and delayed images.) For each run, the following parameters are listed: the calculated exit Reynolds number, $\operatorname{Re}_{\text {exit }}$ (see Eq. (1)), jet pressure ratio, JPR (see Eq. (2)), impingement distance $D_{i m p}$ in $\mathrm{mm}$ and nozzle diameters, measured plenum temperature, $T_{0}$, measured plenum pressure, $p_{0}$, measured ambient pressure in the test chamber, $p_{a}$, and mole fraction of nitric oxide, $\chi_{\mathrm{NO}}$ (given as a percentage). Note that because a $5 \% \mathrm{NO} / 95 \% \mathrm{He}$ mixture was diluted with $100 \% \mathrm{~N}_{2}$, the mole fraction of $\mathrm{He}$ is given by Eq. (12) and the mole fraction of $\mathrm{N}_{2}$ is given by Eq. (13).

$$
\begin{gathered}
\chi_{H e}=19 \cdot \chi_{N O} \\
\chi_{N_{2}}=1-\left(\chi_{H e}+\chi_{N O}\right)
\end{gathered}
$$

We plan to publish a complete appendix as a NASA technical memorandum, which will include the following for each of these 30 runs: a 100-shot averaged, raw (i.e. not dewarped or smoothed) PLIF image; a plot of the measured velocity along the centerline; a pressure profile, with surface pressures from the centerline of the impingement target that have been normalized by the ambient pressure in the test chamber; velocity profiles out to 6 nozzle diameters above and below the jet centerline, as in Fig. 7 (For hardware configuration \#1, there are 15

profiles per run; for hardware configuration \#2, there are 22 profiles per run.); individual velocity profiles for all lines, out to 3 nozzle diameters above and below the jet centerline, as in Fig. 8; a map of the 95\% confidence interval for the run 


\begin{tabular}{|c|c|c|}
\hline \multirow{2}{*}{$\mathbf{x}$} & \multicolumn{2}{|c|}{$\mathbf{y}$} \\
\hline \multirow{2}{*}{$m m$} & upper & lower \\
\cline { 2 - 3 } & $m m$ & $m m$ \\
\hline \hline 0.28 & 16.31 & -16.31 \\
\hline 25.09 & 16.32 & -16.32 \\
\hline 25.92 & 16.23 & -16.23 \\
\hline 26.79 & 16.05 & -16.05 \\
\hline 27.47 & 15.91 & -15.91 \\
\hline 28.02 & 15.79 & -15.79 \\
\hline 29.09 & 15.46 & -15.46 \\
\hline 30.01 & 15.11 & -15.11 \\
\hline 31.50 & 14.38 & -14.38 \\
\hline 32.78 & 13.63 & -13.63 \\
\hline 33.99 & 12.84 & -12.84 \\
\hline 35.45 & 11.52 & -11.52 \\
\hline 36.23 & 10.67 & -10.67 \\
\hline 37.22 & 9.39 & -9.39 \\
\hline 38.09 & 8.13 & -8.13 \\
\hline 38.86 & 6.83 & -6.83 \\
\hline 39.59 & 5.51 & -5.51 \\
\hline 40.31 & 4.28 & -4.28 \\
\hline 41.04 & 3.06 & -3.06 \\
\hline 41.79 & 2.25 & -2.25 \\
\hline 42.76 & 1.58 & -1.58 \\
\hline 43.88 & 1.19 & -1.19 \\
\hline 44.93 & 1.09 & -1.09 \\
\hline 45.86 & 1.21 & -1.21 \\
\hline 46.85 & 1.43 & -1.43 \\
\hline 47.90 & 1.63 & -1.63 \\
\hline 49.01 & 1.78 & -1.78 \\
\hline 50.20 & 1.94 & -1.94 \\
\hline 51.30 & 2.02 & -2.02 \\
\hline 52.46 & 2.08 & -2.08 \\
\hline & & \\
\hline
\end{tabular}

Table A1. Coordinates for supersonic nozzle contour.

\begin{tabular}{|c|c|c|c|c|c|c|c|c|c|}
\hline \multirow{2}{*}{$\begin{array}{c}\text { RUN } \\
\#\end{array}$} & \multirow{2}{*}{$\mathbf{R e}_{\text {exit }}$} & \multirow{2}{*}{$J P R$} & \multicolumn{2}{|c|}{$D_{i m p}$} & \multirow{2}{*}{$\begin{array}{c}T_{0} \\
\kappa\end{array}$} & \multirow{2}{*}{$\begin{array}{c}p_{0} \\
k P a\end{array}$} & \multirow{2}{*}{$\begin{array}{c}p_{a} \\
k P a\end{array}$} & \multirow{2}{*}{$\begin{array}{c}\chi_{\mathrm{NO}} \\
\% \\
\%\end{array}$} & \multirow{2}{*}{$\begin{array}{c}\text { Config } \\
\#\end{array}$} \\
\hline & & & $\mathrm{mm}$ & $\begin{array}{c}\mathrm{Noz} \\
\mathrm{Dia}\end{array}$ & & & & & \\
\hline 397 & 1,201 & 1.8 & 44.5 & 10.7 & 575 & 7.92 & 0.225 & $0.56 \%$ & 1 \\
\hline 399 & 1,221 & 2.2 & 44.5 & 10.7 & 582 & 8.13 & 0.181 & $0.58 \%$ & 1 \\
\hline 401 & 1,013 & 1.7 & 44.5 & 10.7 & 552 & 6.34 & 0.185 & $0.63 \%$ & 1 \\
\hline 403 & 1,989 & 3.6 & 44.5 & 10.7 & 577 & 13.3 & 0.186 & $0.50 \%$ & 1 \\
\hline 405 & 3,828 & 6.2 & 44.5 & 10.7 & 553 & 24.3 & 0.196 & $0.50 \%$ & 1 \\
\hline 407 & 2,036 & 2.9 & 44.5 & 10.7 & 570 & 13.2 & 0.229 & $0.50 \%$ & 1 \\
\hline 409 & 3,862 & 5.5 & 44.5 & 10.7 & 551 & 24.2 & 0.220 & $0.50 \%$ & 1 \\
\hline 411 & 2,918 & 3.9 & 44.5 & 10.7 & 556 & 18.4 & 0.236 & $0.50 \%$ & 1 \\
\hline 413 & 1,236 & 1.6 & 44.5 & 10.7 & 578 & 8.14 & 0.261 & $0.51 \%$ & 1 \\
\hline 415 & 7,249 & 11.6 & 44.5 & 10.7 & 540 & 46.1 & 0.200 & $0.50 \%$ & 1 \\
\hline 417 & 12,049 & 15.6 & 44.5 & 10.7 & 506 & 71.2 & 0.229 & $0.50 \%$ & 1 \\
\hline 419 & 7,379 & 9.1 & 44.5 & 10.7 & 513 & 44.3 & 0.244 & $0.49 \%$ & 1 \\
\hline 421 & 1,444 & 2.1 & 44.5 & 10.7 & 555 & 13.0 & 0.311 & $0.50 \%$ & 1 \\
\hline 423 & 2,328 & 3.9 & 44.5 & 10.7 & 537 & 23.9 & 0.321 & $0.50 \%$ & 1 \\
\hline 425 & 7,542 & 6.9 & 44.5 & 10.7 & 515 & 45.4 & 0.332 & $0.49 \%$ & 1 \\
\hline 427 & 2,561 & 2.9 & 44.5 & 10.7 & 532 & 23.8 & 0.422 & $0.50 \%$ & 1 \\
\hline 429 & 1,292 & 1.6 & 44.5 & 10.7 & 550 & 13.1 & 0.430 & $0.51 \%$ & 1 \\
\hline 431 & 7,564 & 5.2 & 44.5 & 10.7 & 513 & 45.4 & 0.435 & $0.49 \%$ & 1 \\
\hline 433 & 2,524 & 2.0 & 44.5 & 10.7 & 529 & 23.7 & 0.597 & $0.50 \%$ & 1 \\
\hline 435 & 7,541 & 3.5 & 44.5 & 10.7 & 507 & 44.6 & 0.644 & $0.49 \%$ & 1 \\
\hline 504 & 1,117 & 2.3 & 63.5 & 15.2 & 574 & 12.9 & 0.296 & $0.52 \%$ & 2 \\
\hline 506 & 2,168 & 4.2 & 63.5 & 15.2 & 557 & 23.9 & 0.301 & $0.51 \%$ & 2 \\
\hline 508 & 7,305 & 7.6 & 63.5 & 15.2 & 532 & 45.7 & 0.304 & $0.49 \%$ & 2 \\
\hline 510 & 2,418 & 3.0 & 63.5 & 15.2 & 546 & 23.8 & 0.404 & $0.51 \%$ & 2 \\
\hline 512 & 1,191 & 1.6 & 63.5 & 15.2 & 564 & 12.9 & 0.417 & $0.51 \%$ & 2 \\
\hline 514 & 7,413 & 5.2 & 63.5 & 15.2 & 524 & 45.6 & 0.439 & $0.50 \%$ & 2 \\
\hline 516 & 2,374 & 2.1 & 63.5 & 15.2 & 540 & 23.7 & 0.581 & $0.51 \%$ & 2 \\
\hline 518 & 7,463 & 3.6 & 63.5 & 15.2 & 514 & 44.9 & 0.632 & $0.49 \%$ & 2 \\
\hline 520 & 7,393 & 2.6 & 63.5 & 15.2 & 509 & 44.0 & 0.836 & $0.49 \%$ & 2 \\
\hline 522 & 7,361 & 1.8 & 63.5 & 15.2 & 514 & 44.3 & 1.240 & $0.49 \%$ & 2 \\
\hline
\end{tabular}

Table A2. Flow parameters and hardware configuration for velocimetry runs.

\section{Acknowledgements}

The authors wish to acknowledge the collaborative input of Scott Halloran and Don Picetti of The Boeing Company and Chris Glass of NASA Langley Research Center, as well as the assistance of Paul Tucker, also of NASA Langley Research Center. They also would like to thank Chris Ivey of Johns Hopkins University for his invaluable and time-saving assistance in the preparation of this paper. This work was funded as part of the Shuttle Return to Flight effort through Chuck Campbell of Johnson Space Center and Tom Horvath of NASA Langley Research Center. Support was also received from the NASA's Fundamental Aeronautics Program, Hypersonics Project, part of the Experimental Capabilities Discipline. 


\section{References}

${ }^{1}$ Nowak, Robert J., Donald J. Picetti, and Derek S. Liechty, “Tier-II Breaches Jet Impingement Tests, NASA LaRC 31-Inch Mach-10 Tunnel," NASA EG-SS-06-6, 01 November 2006.

${ }^{2}$ Rufer, Shann J., Robert J. Nowak, and Kamran Daryabeigi, "Experimental and Computational Study of Underexpanded Jet Impingement and Heat Transfer," AIAA-2009-4311, 41 ${ }^{\text {st }}$ AIAA Thermophysics Conference, San Antonio, TX, 22-25 June 2009.

${ }^{3}$ Wilkes, Jennifer A., Paul M. Danehy, and Robert J. Nowak "Fluorescence Imaging Study of Transition in Underexpanded Jets," Proceedings of the 21st International Congress on Instrumentation in Aerospace Simulation Facilities (ICIASF) [CDROM], Sendai, Japan, 29 August - 1 September 2005, pp. 1-8.

4 Wilkes, Jennifer A., Christopher E. Glass, Paul M. Danehy, and Robert J. Nowak, "Fluorescence Imaging of Underexpanded Jets and Comparison with CFD," AIAA-2006-0910, 44 $4^{\text {th }}$ AIAA Aerospace Sciences Meeting and Exhibit, Reno, NV, 9-12 January 2006.

${ }^{5}$ Inman, Jennifer A. (Wilkes), Paul M. Danehy, Robert J. Nowak, and David W. Alderfer, "Fluorescence Imaging Study of Impinging Underexpanded Jets," AIAA-2008-619, 46th AIAA Aerospace Sciences Meeting and Exhibit, Reno, NV, 7-10 January 2008.

${ }^{6}$ Inman, Jennifer A. (Wilkes), Paul M. Danehy, Robert J. Nowak, and David W. Alderfer, "Identification of Instability Modes of Transition in Underexpanded Jets," 38th AIAA Fluid Dynamics Conference and Exhibit, Seattle, WA, 23-26 June 2008.

${ }^{7}$ Inman, Jennifer A., Paul M. Danehy, Robert J. Nowak, and David W. Alderfer, "The Effect of Impingement on Transitional Behavior in Underexpanded Jets" AIAA-2009-0591, 47th AIAA Aerospace Sciences Meeting, Orlando, FL, 5-8 January, 2009.

${ }^{8}$ Hsu, Andrea G., Ravi Srinivasan, Rodney D. W. Bowersox, and Simon W. North, "Two-component molecular tagging velocimetry utilizing $\mathrm{NO}$ fluorescence lifetime and $\mathrm{NO}_{2}$ photodissociation techniques in an underexpanded jet flowfield," Applied Optics, 48 (22) 1 August 2009 pp. 4414-4423.

${ }^{9}$ Henderson, Brenda, James Bridges, and Mark Wernet, "An experimental study of the oscillatory flow structure of toneproducing supersonic impinging jets," Journal of Fluid Mechanics, 542, 2005, pp. 115-137.

${ }^{10}$ Dauptain, A, J.B. Harry, G. Staffelbach, A. Roux, B. Cuenot, L.Y.M. Gicquel, and T. Schoenfeld, "Large Eddy Simulation of supersonic impinging jets," CERFACS, Toulouse, France, 2009, to be published.

${ }^{11}$ Bathel, Brett F., Paul M. Danehy, Jennifer A. Inman, Stephen B. Jones, and Christopher B. Ivey, " Multiple Velocity Profile Measurements in Hypersonic Flows Using Sequentially-Imaged Fluorescence Tagging," AIAA-2010-1404, 48th AIAA Aerospace Sciences Meeting, Orlando, FL, 4-7 January 2010. 